\title{
Pólipos em vesícula urinária de um cão - relato de caso
}

\section{Polyp in urinary bladder of a dog - a case report}

\author{
Luciana Curotto Nolasco de Carvalho'; Jaqueline França dos Santos ${ }^{1}$; Monica \\ Vicky Bahr Arias ${ }^{2 *}$; Antônio Carlos Faria dos Reis ${ }^{3}$
}

\begin{abstract}
Resumo
Pólipos são formações benignas raramente encontradas no aparelho urinário tanto na medicina veterinária como na medicina humana. A etiologia ainda não foi identificada, mas acredita-se que ocorra por infecção recorrente do trato urinário, caracterizando uma doença chamada de cistite polipóide. Descreve-se no presente artigo um caso de pólipo vesical em cadela castrada Cocker Spaniel de 11 anos com histórico de hematúria há dois anos. Foi identificada infecção urinária por Serratia spp e ao exame ultrassonográfico observou-se duas massas pedunculadas irregulares em face cranioventral e espessamento da parede vesical. $\mathrm{O}$ animal foi submetido à cistectomia parcial para remoção das massas onde foram observadas várias outras pequenas formações próximas à mesma região. $\mathrm{O}$ exame histopatológico confirmou o diagnóstico de pólipos vesicais. Para o tratamento da doença, além da excisão cirúrgica, a infecção deve ser tratada adequadamente. O prognóstico é bom, porém casos de recidivas podem ocorrer especialmente quando a remoção é feita apenas a partir da mucosa vesical.
\end{abstract}

Palavras-chave: Infecção do trato urinário, pólipo vesical, cães

\begin{abstract}
Polyps are benign formations rarely found in the urinary tract both in veterinary medicine and human medicine. The etiology was not identified, but it is believed to occur by recurrent infection of the urinary tract, characterizing a disease called polypoid cystitis. It was described in this report a case found in a neutered 11 years-old bitch Cocker Spaniel with a history of hematúria for two years. It was identified a urinary infection by Serratia spp and the ultrassond examination showed two irregular pedunculated masses in the cranioventral portion and thickening of the bladder wall. The animal was underwent parcial cystectomy to remove the masses where it was observed other small formations near the same region; the material was referred to histopathological examination to confirm the diagnosis of bladder polyps. For treatment of disease, in addition to surgical excision, the infection should be treated properly. The prognosis is good, but cases of recurrence may occur especially when the removal is made only from the bladder mucosa.
\end{abstract}

Key words: Urinary tract infection, bladder polyps, dogs

\section{Introdução}

Pólipos são massas não neoplásicas que se assemelham a tumores e são sequelas de inflamação crônica, sendo sua ocorrência descrita nas espécies equina e felina e com menor intensidade em outras espécies (THOMSON, 1990). São de origem epitelial e classificados histologicamente como pólipo fibroepitelial (PATRICK et al., 2006). O

\footnotetext{
1 Pós-Graduando, Dept ${ }^{\circ}$ de Clínicas Veterinárias, Universidade Estadual de Londrina, UEL, Londrina, PR. E-mail: lulucarv@ hotmail.com; jaquejfs@yahoo.com.br

2 Prof ${ }^{a}$ Associada, Dept ${ }^{\circ}$ de Clínicas Veterinárias, UEL, Londrina, PR. E-mail: vicky@uel.br

3 Prof. Adjunto, Dept ${ }^{\mathrm{o}}$ de Medicina Veterinária Preventiva, UEL, Londrina, PR. E-mail: reis@uel.br

*Autor para correspondência
} 
pólipo fibroepitelial é uma lesão que se manifesta macroscopicamente como uma pequena massa mole e vascularizada de tecido conjuntivo localizado abaixo da mucosa, projetando-se para a luz do órgão (COTRAN; KUMAR; ROBBINS, 1996).

Em humanos o pólipo fibroepitelial ocorre mais comumente em ureter e pode aparecer também na bexiga, pelve renal e uretra (COTRAN; KUMAR; ROBBINS, 1996). Na medicina veterinária, são mais comumente encontrados em região de ouvido médio e nasofaríngea de gatos e nasal e etmoidal de equinos (THOMSON, 1990). Pólipos na vesícula urinária de cães caracterizam uma doença rara chamada cistite polipóide havendo inflamação, proliferação epitelial e desenvolvimento de massas polipóides sem evidência neoplásica (MARTINEZ et al., 2003).

A etiologia da cistite polipóide é desconhecida, provavelmente relacionada à inflamação ou irritação persistente da mucosa vesical. Isto é descrito em humanos cateterizados por longos períodos (MARTINEZ et al., 2003; FARRELL; PHILBEY; RAMSEY, 2006). A etiologia da doença pode estar relacionada a defeitos do desenvolvimento, visto a afecção ser encontrada em neonatos. Trauma, obstrução e desequilíbrio hormonal são outros possíveis fatores causais (FARRELL; PHILBEY; RAMSEY, 2006).

Martinez et al. (2003) descreveram 17 casos de cistite polipóide em cães, em um período de 23 anos, sendo a maioria fêmeas que apresentavam hematúria e infecção de trato urinário recorrente.

O objetivo deste trabalho é relatar esta afecção em uma cadela de 11 anos de idade com histórico de hematúria crônica, infecção de trato urinário e massas múltiplas em mucosa de vesícula urinária.

\section{Relato do Caso}

Foi atendida no Hospital Veterinário da Universidade Estadual de Londrina uma cadela Cocker Spaniel Americano de onze anos de idade, de 12 quilos, com histórico de hematúria no início da micção e coágulos de sangue na urina, cujos episódios ocorriam intermitentemente há dois anos. $\mathrm{O}$ animal havia sido submetido à mastectomia e ovariosalpingohisterectomia há três anos devido a presença de nódulos mamários, cujo laudo histopatológico foi carcinoma complexo.

Na urinálise realizada na paciente foi identificada urina de cor avermelhada, turva, com densidade de 1,015. As leituras de $\mathrm{pH}$, acetona, proteína, glicose, bilirrubina e sangue não foram possíveis devido a interferência de cor. No exame do sedimento urinário a contagem de leucócitos foi de 600 por campo e de 1240 hemáceas por campo, três cruzes de cristais de fosfato amorfo e também três cruzes de bactérias. Na urocultura detectou-se infecção urinária por Serratia spp.

No exame ultrassonográfico de abdômen foram identificadas na vesícula urinária duas massas irregulares, pedunculadas de base estreita medindo aproximadamente $1,8 \times 1,6 \mathrm{~cm}$, com espessamento da parede vesical ventral que media cerca de $0,52 \mathrm{~cm}$.

Nos exames hematológicos e bioquímicos préoperatórios não foram encontradas alterações. Foi realizada cirurgia de cistotomia, confirmando-se a presença de uma massa de cerca de $3 \mathrm{~cm}$ de diâmetro e múltiplos nódulos verrucosos semelhantes na parede do ápice vesical, realizando-se então uma cistectomia parcial de um quarto da parede vesical para remoção das lesões (Figura 1). De acordo com o resultado da urocultura e antibiograma, o animal foi tratado no pós-operatório com Sulfametoxazol + Trimetoprina na dose de $15 \mathrm{mg} / \mathrm{Kg}$ a cada 12 horas, durante 21 dias. Houve resistência antimicrobiana a outros antibióticos testados como amoxacilina, ampicilina e cefalotina e sensibilidade à sulfametoxazol + trimetoprina, norfloxacina, enrofloxacina e nitrofurantoína.

Ao exame histopatológico das neoformações em bexiga foram identificadas poucas figuras de mitose no epitélio hiperplásico, com formações papilares e proliferação multifocal de células uroteliais 
vacuolizadas, além de proliferação de vasos sanguíneos com hemorragia difusa na lâmina própria e presença de células inflamatórias como linfócitos e neutrófilos. Foram encontrados ainda infiltrados linfóides na camada muscular hipertrofiada, microabscessos no córion e aglomerados de epitélio com abertura central (Figura 2). Estas alterações permitiram classificar as massas como pólipo vesical.

Figura 1. A - Espessamento da parede vesical. B - Visualização dos pólipos em mucosa vesical, após a cistotomia.

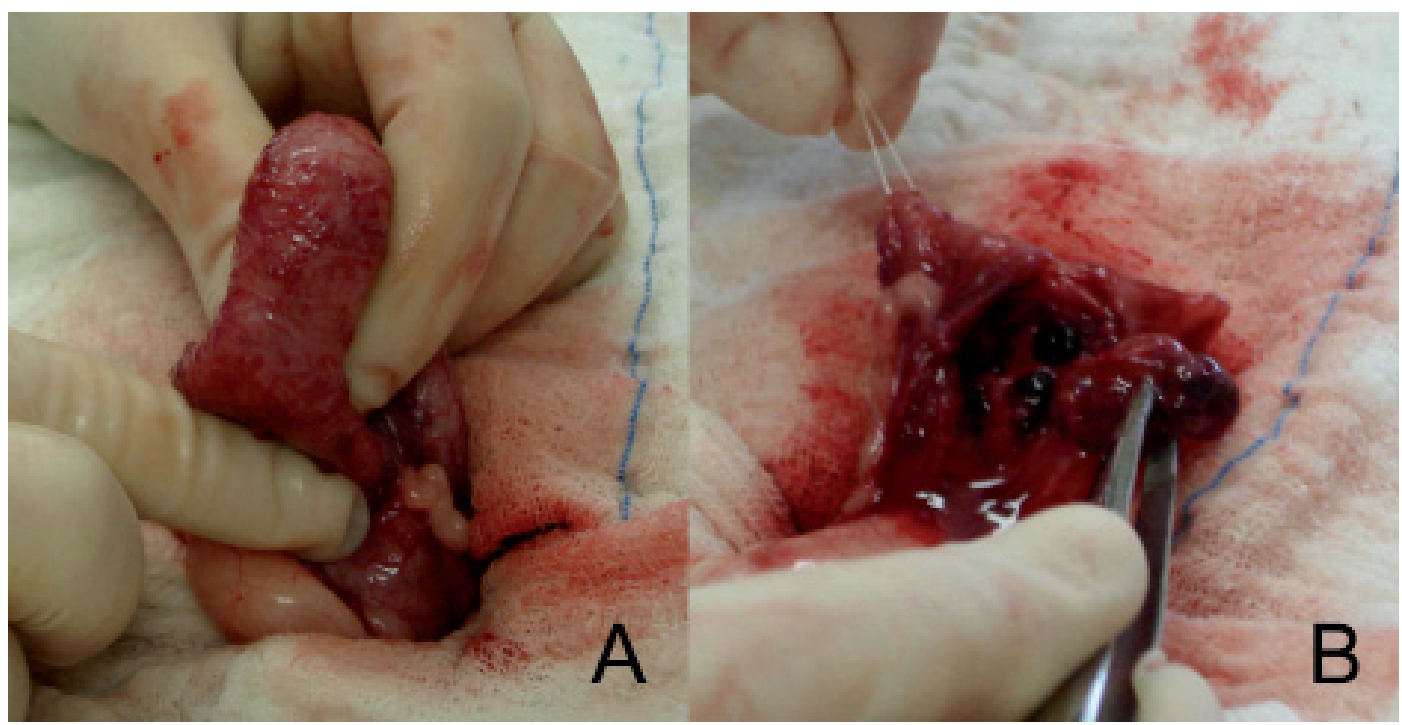

Figura 2. Hiperplasia do epitélio de mucosa vesical com congestão e hemorragia estromal, acompanhado de infiltrado inflamatório. HE; 10X.

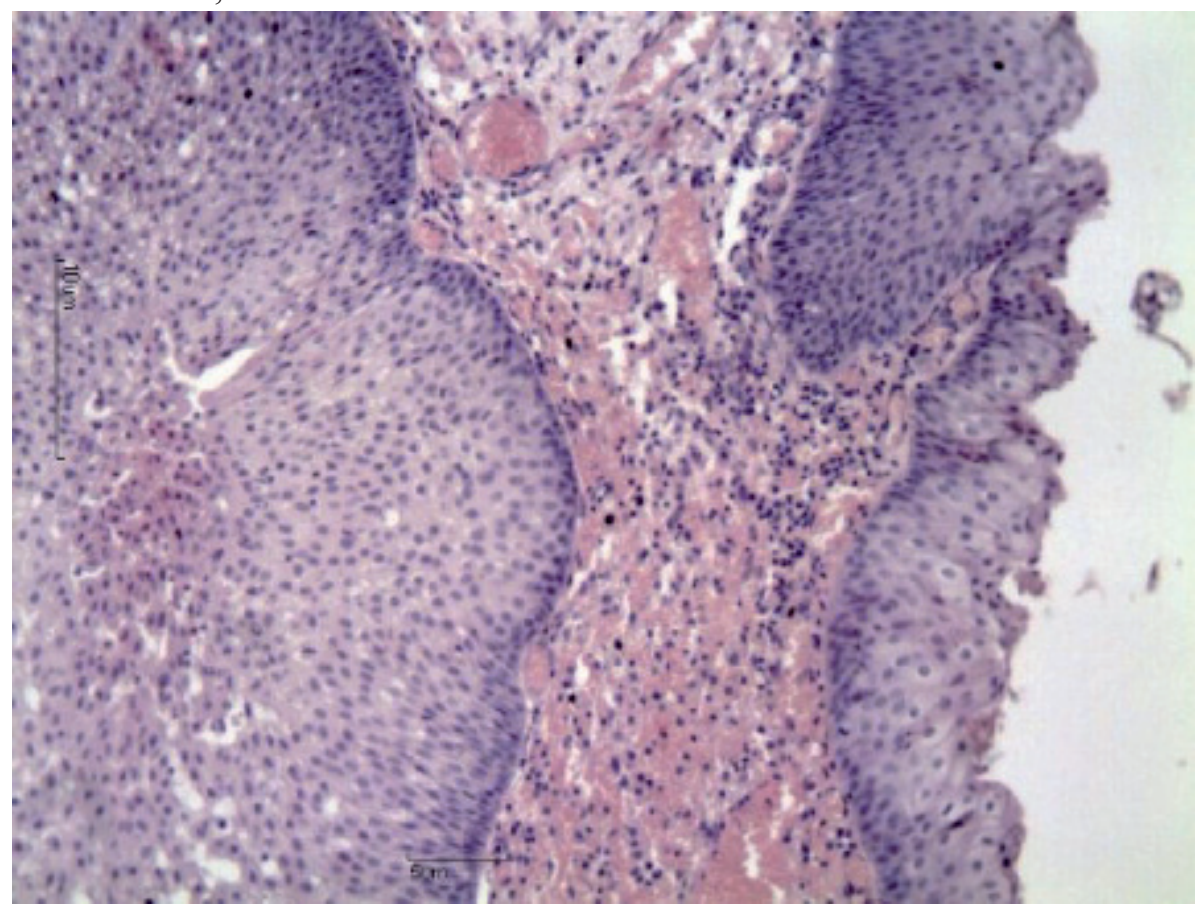


O paciente foi acompanhado por 285 dias após a cistectomia, não apresentando mais hematúria e/ ou sinais clínicos de infecção urinária, enquanto que em novo exame ultrassonográfico realizado não foram encontradas alterações em parede vesical.

\section{Discussão}

No presente caso, o paciente era uma fêmea de 11 anos com histórico de infecção crônica do trato urinário sem cálculo vesical. De acordo com levantamento de 17 casos de cistite polipóide em cães realizado por Martinez et al. (2003), as fêmeas com idade média de sete anos são mais afetadas. A irritação local da mucosa que leva à formação dos pólipos pode estar associada à infecção ou cálculo vesical. Neste mesmo levantamento, dos 17 animais, sete apresentavam cálculos, sendo que seis com urocultura positiva e um sem infecção associada ao cálculo e aos pólipos. A irritação da mucosa pode ser também causada por ação de urólitos sem infecção urinária associada (MARTINEZ et al., 2003). Não se pode afirmar que a infecção urinária seja a responsável pela formação dos pólipos, visto que estes também podem predispor à infecção recorrente, por abrigarem bactérias em locais inacessíveis à ação dos antibióticos (MARTINEZ et al., 2003).

Os sinais clínicos mais comumente descritos são hematúria e histórico de infecção recidivante do trato urinário, como encontrado no presente caso. Outros sintomas que podem ser observados são polaquiúria, estrangúria, dor abdominal, poliúria e polidipsia, noctúria, secreção vaginal, eliminação urinária inapropriada e lambedura excessiva da vulva (MARTINEZ et al., 2003; TAKIGUSHI; INABA, 2005).

$\mathrm{Na}$ urinálise realizada neste caso, identificou-se grave infecção de trato urinário inferior, conforme também notado em urinálises realizadas nos cães estudados por Martinez et al. (2003), que verificaram piúria em oito dos 16 pacientes avaliados, hematúria em 13 dos 16 e bacteriúria em 10 dos 16 pacientes.

Exames ultrassonográficos ou radiografias contrastadas são importantes ferramentas para auxiliar no diagnóstico da cistite polipóide, o que foi realizado neste caso. Takigushi e Inaba (2005) relataram em oito cães com cistite polipóide, seis machos e duas fêmeas, massas polipóides e pedunculadas na bexiga identificadas através do ultrassom. Na maioria dos pacientes as lesões eram múltiplas e a ecogenicidade das massas era isoecóica quando comparadas à parede vesical, porém em alguns casos encontraram-se focos hipoecóicos no interior das massas. Ao exame radiográfico contrastado, essas neoformações foram identificadas como falhas irregulares na porção cranial da bexiga. Nos dois tipos de exames pode ser difícil diferenciar pólipos de coágulos de sangue. No presente relato foi realizada radiografia simples para descartar a presença de cálculo radiopaco e a seguir optou-se pela realização de exame ultrassonográfico, menos invasivo que o exame radiográfico contrastado, sendo possível a visualização das massas.

No presente relato, as massas foram identificadas em região do ápice da bexiga, conforme citado por Takigushi e Inaba (2005). É importante lembrar que neoplasias malignas, como o carcinoma de células transicionais, são encontradas mais comumente na região do trígono vesical(WITHROW, MACEWEN, 2001). Macroscopicamente as lesões são descritas como formações polipóides vascularizadas e macias projetando-se para a luz da bexiga (MARTINEZ et al., 2003), como observado no transoperatório.

No exame histopatológico, os autores Martinez et al. (2003) e Cotran, Kumar e Robbins (1996) descreveram proliferação de tecido conjuntivo abaixo da mucosa com infiltrados de leucócitos mononucleares com ou sem participação de neutrófilos e hemorragia estromal ou acúmulo de hemossiderina, como constatado no presente caso. O exame histopatológico é essencial para diferenciar de neoplasias como o tumor de células transicionais, que pode inclusive ocorrer simultaneamente aos 
pólipos e possui prognóstico e tratamento distintos da cistite polipóide (MARTINEZ et al., 2003; TAKIGUSHI; INABA, 2005).

O tratamento indicado em medicina veterinária é a excisão das massas através de cistotomia ou cistectomia parcial (MARTINEZ et al., 2003) e pode ser curativo. $O$ tratamento de escolha em medicina humana é a ressecção através de cistoscopia e o prognóstico é excelente, sem relato de recorrências (KUMAR et al., 2008).

Rawlings et al. (2007) descreveram o uso de laparoscopia assistida por cistoscopia para a remoção de pólipos vesicais em dois cães, onde foi possível remoção das massas com margem cirúrgica segura. Johnston, Osborne e Stevens (1975), descreveram recidiva em cão quando realizada excisão dos pólipos utilizando o eletrocautério, porém o mesmo não ocorreu em outro caso relatado pelos autores quando realizou-se a excisão por cistectomia parcial de uma grande massa somado à excisão de outras duas massas menores removendo-as a partir da mucosa e submucosa.

Após a excisão das formações deve ser feito tratamento adequado da infecção urinária baseado em cultura e antibiograma seriados. Em estudo realizado por Martinez et al. (2003), a infecção por Proteus spp. foi mais comumente encontrada, seguido de Escherichia coli, Sthaphylococcus spp. e Enterococcus spp. No presente relato a bactéria identificada foi Serratia spp., não referida neste estudo. Infecções por Serratia spp. são raras e são pouco descritas na literatura humana e/ou veterinária. É encontrada em infecções hospitalares causando infecções de trato urinário, de trato respiratório e cateteres intravenosos (MENEZES et al., 2004). $O$ resultado do antibiograma, com sensibilidade a mais de uma classe de antibiótico, leva a crer que a infecção não era de origem hospitalar.

No presente relato a antibioticoterapia instituída foi adequada para a eliminação da infecção e a cistectomia parcial foi curativa não apresentando recidiva dos sinais clínicos e ultrasonográficos até o $285^{\circ}$ dia após sua realização.

Pólipos vesicais são raros e não possuem origem determinada, porém devem ser incluídos no diagnóstico diferencial de casos de hematúria crônica e/ou infecção recidivante de trato urinário. O tratamento cirúrgico é curativo, porém a infecção urinária deve ser tratada e controles pós-operatório com radiografias contrastadas ou ultrassonografias devem ser realizadas.

\section{Referências}

COTRAN, R. S.; KUMAR, V.; ROBBINS, S. L. Patologia estrutural e funcional. 5. ed. Rio de Janeiro: Guanabara Koogan, 1996.

FARRELL, M.; PHILBEY, A. W.; RAMSEY, I. Ureteral fibroepithelial polyp in a dog. Journal of Small Animal Practice, Gloucester, v. 47, n. 7, p. 409-412, 2006.

JOHNSTON, S. D.; OSBORNE, C. A.; STEVENS, J. B. Canine polypoid cistitis. Journal of the American Veterinary Medical Association, Schaumburg, v. 116, n. 12, p. 1155-60, 1975.

KUMAR, A.; DAS, S. K.; TRIVEDI, S.; DWIVEDI, U. S.; SINGH, P. B. Genito-urinary polyps: summary of the 10-year experiences of a single institute. International Urology and Nephrology, Toronto, v. 40, n. 4, p. 901-907, 2008.

MARTINEZ, I.; MATTOON, J. S.; EATON, K. A.; CHEW, D. J.; DIBARTOLA, S. P. Polypoid cystitis in 17 dogs (1978-2001). Journal of Veterinary Internal Medicine, Lakewood, v. 17, n. 4, p. 499-509, 2003.

MENEZES, E. A.; CEZAFAR, F. C.; ANDRADE, M. S. S.; ROCHA, M. V. A. P.; CUNHA, F. A. Frequency of Serratia sp in urine infections of intern patients in the Santa Casa de Misericórdia in Fortaleza, Revista da Sociedade Brasileira de Medicina Tropical, Uberaba, v. 37, n. 1, p. 70-71, 2004.

PATRICK, D. J.; FITZGERALD, S. D.; SESTERHENN, I. A.; DAVIS, C. J.; KIUPEL, M. Classification of canine urinary bladder urothelial tumours based on the world health organization/international society of urological pathology consensus classification. Journal of Comparative Pathology, Bristol, v. 135, n. 4, p. 190-199, 2006.

RAWLINGS, C. A. Ressection of inflammatory polyps in dogs using laparoscopic - assisted cystoscopy. Journal of the American Animal Hospital Association, Denver, v. 
43, n. 6, p. 342-46, 2007.

TAKIGUSHI, M.; INABA, M. Diagnostic ultrasound of polypoid cystitis in dogs. Journal of Veterinary Medical Science, Tokio, v. 67, n. 1, p. 57-61, 2005.

THOMSON, R. G. Patologia veterinária especial. São Paulo: Manole, 1990.

WITHROW, S. J.; MACEWEN, E. G. Small animal clinical oncology. 3. ed. Philadelphia: Saunders Elsevier, 2001. 